Musées, Patrimoine et Culture scientifiques et techniques

$123 \mid 2009$

mai - juin 2009

\title{
La maquette entre reconstitution savante et récit imaginaire dans les expositions archéologiques
}

\section{Daniel Jacobi}

\section{(2) OpenEdition \\ Journals}

Édition électronique

URL : http://journals.openedition.org/ocim/234

DOI : 10.4000/ocim.234

ISSN : 2108-646X

Éditeur

OCIM

Édition imprimée

Date de publication : 1 mai 2009

Pagination : $15-23$

ISSN : 0994-1908

Référence électronique

Daniel Jacobi, « La maquette entre reconstitution savante et récit imaginaire dans les expositions archéologiques ", La Lettre de l'OCIM [En ligne], 123 | 2009, mis en ligne le 01 mai 2011, consulté le 19 avril 2019. URL : http://journals.openedition.org/ocim/234 ; DOI : 10.4000/ocim.234 
L'exposition utilise toute une série de registres sémiotiques. D'abord, en tant que support pour présenter des pièces remarquables tirées des collections, ellesmêmes issues de fouilles ou d'autres recherches archéologiques. Mais aussi pour concevoir des artefacts qui matérialisent les résultats plus théoriques et donc plus abstraits des recherches archéologiques.

C'est cette mutation qui a sans doute permis à l'imaginaire de trouver sa voie au sein du discours muséographique propre à l'archéologie. Dorénavant, cohabitent au sein des expositions d'archéologie deux grandes directions complémentaires. D'un côté, les objets archéologiques authentiques mis au jour fortuitement ou extraits et découverts lors de fouilles systématiques. Ils constituent des documents et des preuves qui attestent de la présence ancienne de peuples ou de civilisations disparues. Ou encore affinent et complètent des données connues. Ou confirment localement des découvertes uniques.

D'un autre côté, des dispositifs et toutes sortes d'artefacts conçus pour transposer au plan muséographique les idées, les connaissances ou les savoirs des scientifiques. Ces dispositifs proprement communicationnels, inventés et construits pour les besoins de la cause - et de l'exposition proprement dite -, sont expressément destinés, soit à susciter et provoquer chez les visiteurs des sensations, des émotions, du plaisir ou des sentiments, soit à leur permettre de s'approprier des connaissances et des savoirs archéologiques.

\section{Entre science et exhibition}

S’il est vrai que les musées archéologiques sont restés un peu en retrait de la transformation rapide de l'univers muséal (les musées de sciences, puis les musées des beaux-arts ont ouvert la voie), tel n'est plus le cas aujourd'hui et des grands noms de l'architecture y contribuent notablement à l'exemple de Norman Foster - l'architecte du Carré d'art de Nîmes - pour le musée préhistorique de Quinson. On peut citer aussi la manière dont a été modernisée la muséographie de Saint-Romain-en-Gal. Remarquable est aussi le travail qu'un élève de Le Corbusier, spécialiste d'équipement muséographique : Henri Ciriani (l'architecte, entre autres, de l'Historial de Péronne) a mené à Arles, à proximité des fouilles du cirque romain, en construisant et en aménageant le musée départemental Arles Antique sous la forme d'un triangle de béton revêtu de vitres d'un bleu intense.

L'intervention de grands architectes contemporains fait basculer le musée dans l'univers des constructions qui se remarquent et qui sont faites aussi pour devenir des sortes de signes-symboles d'une ville ou d'une époque. Un architecte, parce qu'il cherche à imprimer son geste et son style, ne saurait se contenter de signer un projet conventionnel de bâtisse anonyme mais parfaitement fonctionnelle. Dorénavant un musée est aussi un monument emblématique de la culture contemporaine au point de se demander si les collections sont mises en valeur par le monument moderne ou si elles ne sont qu'un fairevaloir d'une brillante enveloppe architecturale.

Contrairement au projet de George-Henri Rivière qui faisait du musée et de l'exposition une sorte de parangon et d'aboutissement de la recherche scientifique, l'imaginaire demeure au coeur de ce dispositif communicationnel, qu'il s'agisse, d'un côté, de la conception architecturale et muséographique, de l'élaboration de dispositifs muséographiques singuliers, et de l'autre côté, des modalités de reconnaissance de l'exposition par les visiteurs. Cependant, une des difficultés majeures qui contribue à ne pas laisser l'imagination prendre le pouvoir est l'instauration d'un système de règles implicites qui de plus en plus surdétermine la conception des expositions.

\section{L'exposition comme système de conventions implicites}

Comment rendre compte d'une observation qui frappe tout observateur qui parcourt les musées archéologiques en Europe : à savoir que leurs expositions se ressemblent toutes et qu'en dépit de la variété de leurs collections, elles ont entre elles comme un air de famille ? C'est sans doute que la muséographie, en dépit des déclarations de liberté et de créativité de ceux qui la conçoivent obéit à des formes et choisit des dispositifs canoniques qui sont autant de conventions expographiques imposées aux scientifiques conservateurs.

Par exemple, la datation est une impérieuse nécessité. On se doit en, conséquence, de respecter strictement la stratigraphie. Les couches successives de dépôt imposent une lecture des archives unidirectionnelle du bas vers le haut : plus la couche productive est profonde, plus elle est ancienne. Plus elle est proche de la surface du sol contemporain, plus elle est récente. Ce mode de lecture - du bas vers le haut - a beau être aberrant et illogique pour des visiteurs qui ont automatisé la lecture du haut vers le bas (comme elle l'est de la gauche vers la droite), aucun spécialiste ne peut se permettre d'y déroger.

Autre convention rigide, une présentation ne saurait contredire la chronologie. Et tant pis si la protohistoire impose de donner à voir d'abord des traces 
préhistoriques communes et banales (silex, bivalves, haches, os) que l'on rencontre dans tous les musées d'archéologie ou, un peu plus loin dans le parcours, des débris de poterie dont seuls quelques fragments sont chichement décorés. Pour exposer les chefs d'œuvres et les pièces les plus remarquables, le parcours attendra que la période la plus riche et la plus fertile vienne à sa place dans un parcours scrupuleusement chronologique.

Sans parler des effets de saturation et des redoublements qui ont des effets déplorables au plan de l'attractivité. Il convient d'exposer toutes les pièces remarquables trouvées sur place ou dans la région et non pas une seule. Et tant pis si les objets encombrants (fours de potiers et épaves de bateaux antiques à Marseille ou ailleurs stock d'amphores) viennent encombrer l'espace et se faire de l'ombre les uns aux autres. Le goût pour les séries, probablement hérité de la tradition des muséums d'Histoire naturelle, tout comme le sont l'attrait pour les classifications, les taxonomies ou la volonté de faire apparaître l'évolution, expliquent sans doute pourquoi les documents archéologiques étaient disposés dans des vitrines dans lesquelles débris ou objets étaient disposés en longs alignements monotones. L'accumulation qui voulait signifier la richesse du site secrétait bien souvent la saturation et l'ennui. Ainsi l'exposition archéologique n'était-elle trop souvent qu'un ensemble de litanies monotones et ennuyeuses que seuls les spécialistes pouvaient déchiffrer, non sans recourir aux petites étiquettes qui accompagnent inévitablement chacun de ces échantillons tous datés et localisés.

A contrario, les objets archéologiques les plus remarquables (belles pièces ou trouvailles) ont été longtemps muséographiés à la manière des œuvres d'art. Présentés dans des vitrines de verre, éclairés savamment et disposés devant des cimaises colorées pour faire ressortir par contraste les qualités esthétiques de ces pièces remarquables, ils évoquaient irrésistiblement la muséographie propre aux beaux arts. Certes, la nature de la pièce et la période remplaçaient le titre de l'œuvre (sculpture ou tableau) tandis que le nom de l'inventeur était substitué à celui de l'artiste, le lieu et la date de la découverte figurant en lieu et place des techniques utilisées par l'artiste. Mais, il est assez évident que le style de mise en exposition tout comme l'effet que l'on cherchait à produire sur le visiteur reflétait l'idéologie propre au «bon » goût, qui stipule, en France en tout cas, que l'on puisse goûter et admirer une chose sans qu'il soit nécessaire de la comprendre ni donc de l'expliciter.

\section{De l'interprétation scientifique à l'imaginaire expographique : le cas des maquettes}

Quelles sont les ressources ou plutôt les ressorts muséographiques qui peuvent permettre de reconsidérer ce type de mise en exposition ? On aurait tort de penser que la recherche archéologique n'est qu'une science froide et inhumaine. L'archéologue qui tente de reconstituer un monde disparu à partir d'un certain nombre de traces lacunaires et en trop petit nombre doit puiser dans son imagination pour remplacer toutes les pièces manquantes d'un puzzle dont on ne dispose que de quelques pièces sans savoir de plus si elles sont primordiales.

L'un des ressorts essentiels de cette quête difficile et aventureuse qu'est toute reconstitution lorsqu'on ne dispose pas de sources documentaires écrites qui peuvent témoigner et attester de ce qu'était le monde disparu est de procéder par comparaison avec la vie contemporaine ou par analogie avec des civilisations archaïques déjà connues et décrites par des sources attestées.

Du coup, il devient assez plausible de sonoriser (dans un dispositif d'audio-guidage) une scène de festin romain à l'aide de chants braillards de convives enivrés alternant avec la musique des aèdes et de leurs lyres sur un fond de bruits d'assiettes et de

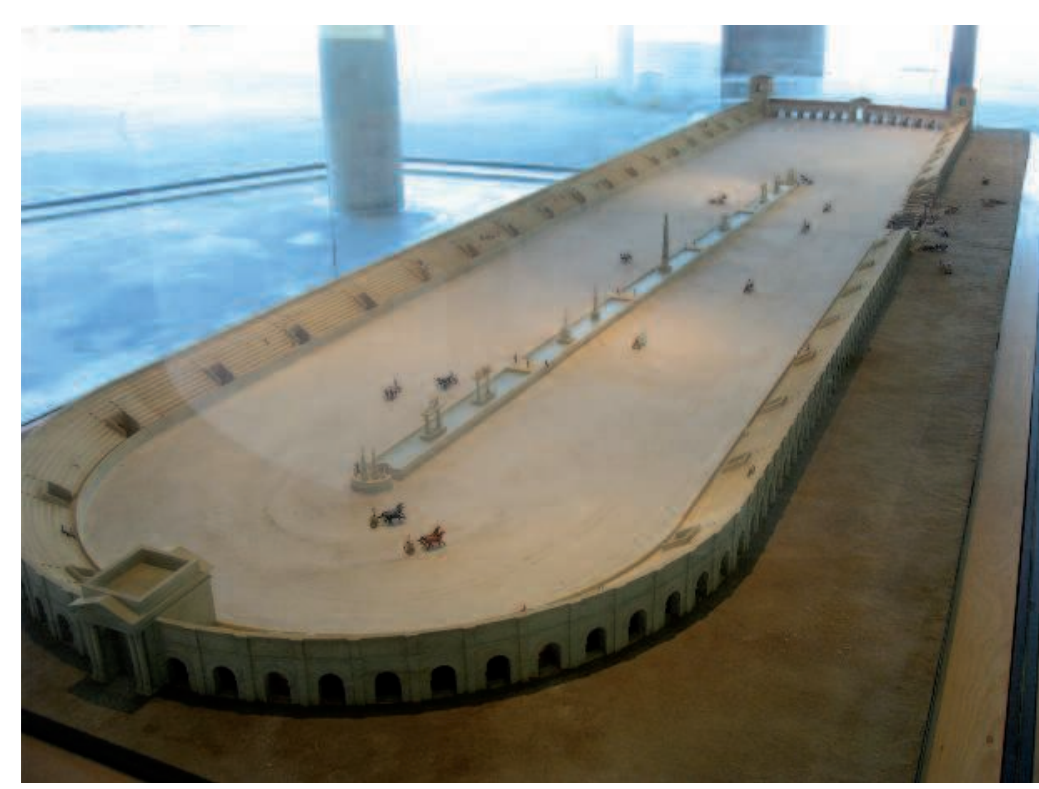

Le cirque : la maquette du cirque est le seul élément permettant $d$ 'interpréter les vestiges archéologiques que I'on aperçoit à travers les larges baies vitrées du musée

(maquette au 1/100e, conçue par les musées d'Arles et Patrice Berger avec l'aide et les conseils de G. Hallier, CNRS et de J. Humphrey, Ann Arbor University). (c) Daniel Jacobi 


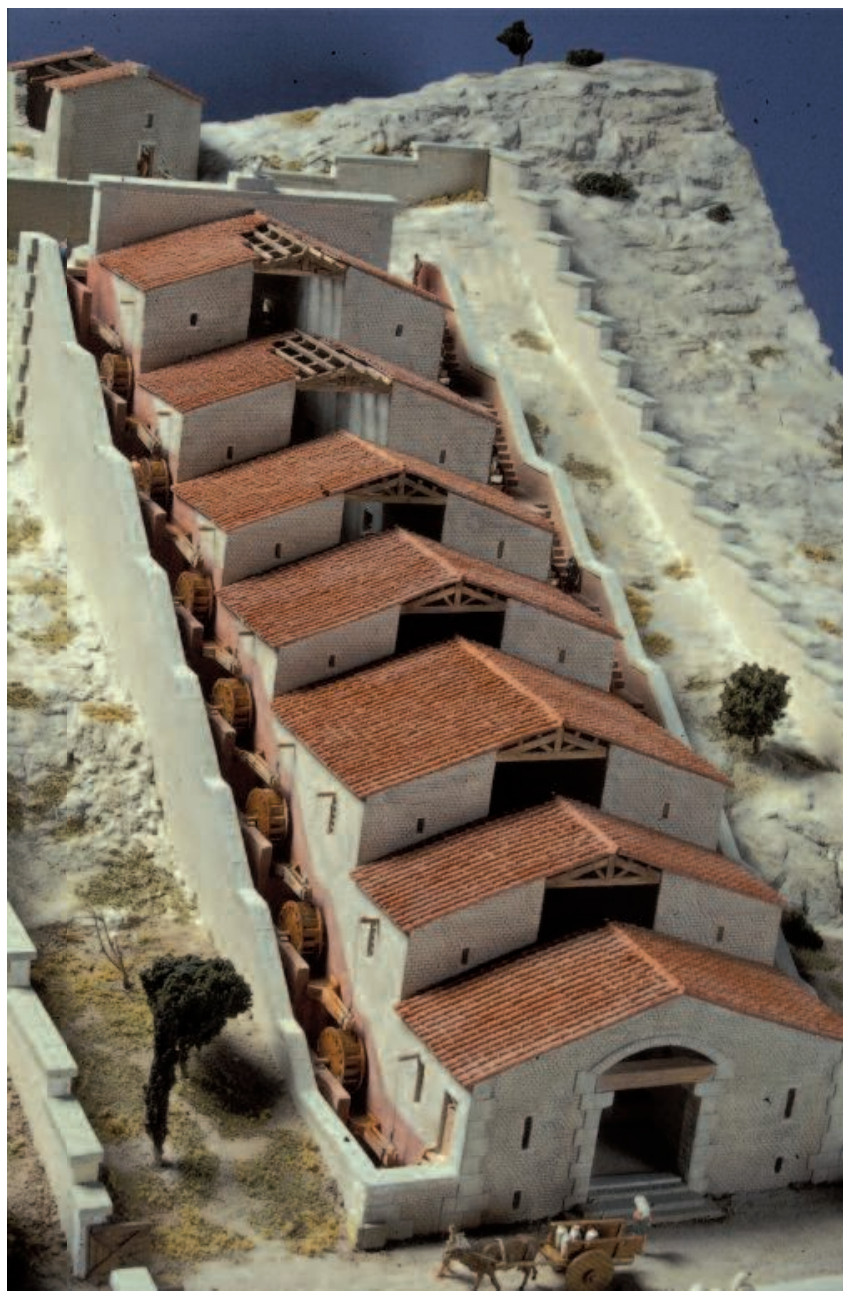

La meunerie hydraulique industrielle de Barbegal à la fin du Haut-Empire : avec cette maquette, le musée parvient à donner vie à un site archéologique distant, aujourd'hui complètement détruit et difficile à déchiffrer (détail de la maquette au 1/100e, conçue par J.-L. Paillet, CNRS et réalisée par Denis Delpallilo). ๑) Musée d'Archéologie antique d'Arles/M. Lacanaud

couverts. Le trésor découvert dans un dépôt peut être présenté comme dans une vitrine de magasin avec des mannequins pour valoriser les colliers d'or et les bagues ornées de pierres précieuses qu'il renfermait (musée archéologique, Dijon). Et il est logique de demander à un décorateur de théâtre de dessiner le paysage (celui d'un cimetière à la tombée du jour) pour muséographier les couvercles de sarcophages et les pièces trouvées dans les tombes d'une nécropole gallo-romaine (musée gallo-romain, Nuits-Saint-Georges).

On sait que par jeu, par défi scientifique et à fins d'administration de la preuve ou encore à des fins pédagogiques, on n'a pas hésité à faire appel à des acteurs qui, ici allument un feu sans allumette, là taillent du silex. D'autres cuisinent ou dégustent des plats romains, ou encore se battent comme des gladiateurs et disputent des concours olympiques grecs et ainsi de suite. Le cinéma (le péplum, le film préhistorique ou le docu-fiction du genre Odyssée de l'espèce) et les bandes dessinées ne sont plus très éloignées du musée. Il était à peu près inévitable que les professionnels de la fiction (pour des spectacles destinés à distraire le public), proposent leur service aux responsables des expositions scientifiques sérieuses.

Cependant l'une des ressources les plus communes de ce recours à l'imaginaire et à l'interprétation (même si dans un musée elle se veut scientifique, intellectuelle et sérieuse, motivée par des preuves et ainsi de suite) est le recours à des maquettes. La maquette est une reproduction à l'identique d'une construction ou d'un site, c'est-à-dire une copie reproduite fidèlement mais à une échelle réduite. Construite avec des moyens rudimentaires, colorée et parfois légendée, elle est aussi très souvent animée par des petits personnages. Rappelons que leur présence est officiellement justifiée par des considérations de lisibilité de la maquette : la taille des sujets humains permettrait au regardeur de reconstituer les dimensions réelles de la construction montrée en réduction. C'est évidemment la présence de ces petits actants-personnages colorés et costumés qui confère à la maquette son attractivité et sa dimension irrémédiablement narrative. Les petits sujets, comme dans la crèche de Noël ou un jeu de Lego, focalisent l'attention des visiteurs. Leur présence humanise la scène. Elle active la projection personnelle et identificatoire des visiteurs ( "ils étaient comme nous ») et le repérage des gestes et des postures contribue à fictionnaliser l'instantanéité de la scène montrée.

La maquette du Pont de bateaux de l'époque romaine visible au MDAA (Arles) est bien représentative du genre maquette. En même temps qu'elle donne à voir un (hypothétique ?) système technique et concrétise les sources écrites décrivant ce pont et sa situation sur le fleuve, la maquette reconstitue une scène quotidienne dans laquelle des actants animés (bateaux, charrettes, employés qui relèvent la partie mobile du pont, soldats...) viennent raconter et illustrer un moment de la vie quotidienne dans l'Antiquité. Et cette analogie saisissante avec une scène quotidienne contemporaine, par sa puissance évocatrice, est directement reconnue, interprétée et racontée par les visiteurs, quelle que soit leur culture technique ou historique préalable. 
La maquette de l'Amphithéâtre (toujours à Arles) est probablement l'une des mieux réussies. Son élaboration, d'une finesse et d'un soin très recherchés, condense une série considérable d'informations scientifiques. En effet, la maquette ne se contente pas de reproduire à l'identique - une échelle convenable - les formes actuelles des arènes d'Arles pourtant assez bien conservées et régulièrement réparées. Cinq catégories d'informations sont ajoutées à la représentation des formes architecturales du monument (informations qu'aucun des visiteurs du monument sur place ne peut percevoir en visitant le «vrai » monument) :

- la maquette permet de reconstituer les parties détruites et disparues, comme par exemple le système de vélum amovible qui permettait d'abriter l'amphithéâtre du soleil (et de la pluie ?) ;

- un éventré (système conventionnel qui propose de montrer l'intérieur d'une construction habituellement non visible à côté de ce qui est visible de l'extérieur) permet de donner à voir les circulations sous les gradins et le système de construction de ces derniers ;

- à la périphérie de la maquette de l'amphithéâtre, un autre éventré fait apparaître les modalités de construction du monument : échafaudage, engins de levage, taille des pierres et esclaves au travail ;

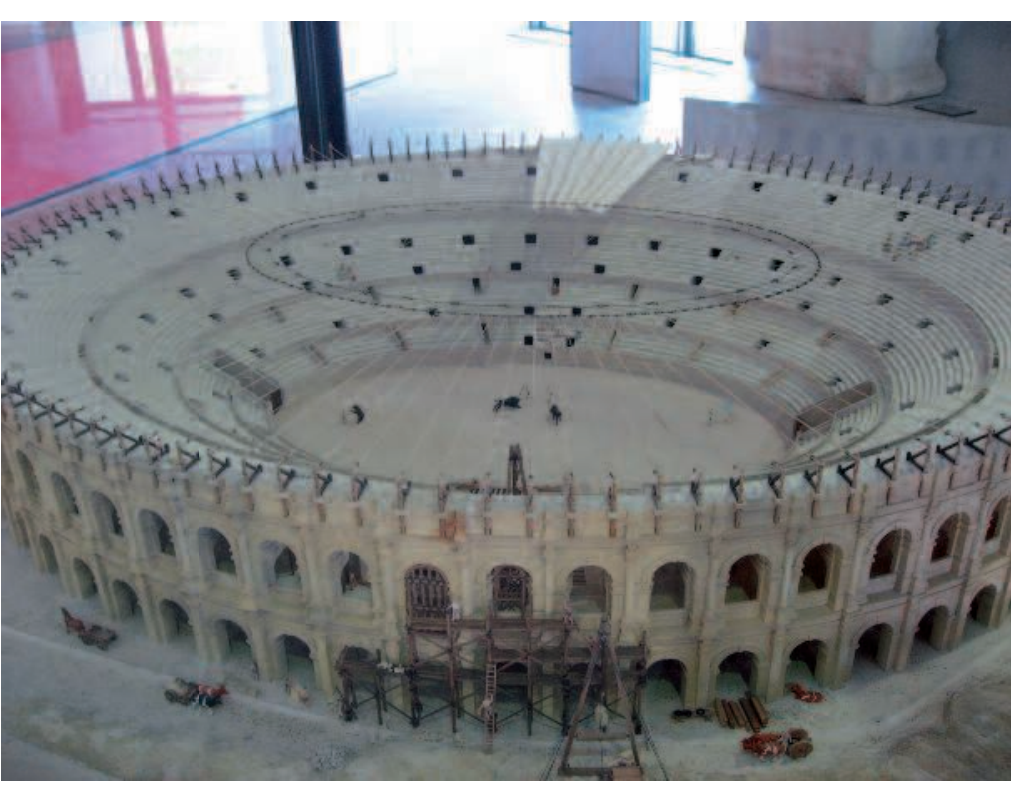

L'amphithéâtre d'Arles à l'époque flavienne : la maquette de l'amphithéâtre est très élégante. Elle reconstitue, non seulement les équipements dont bénéficiait cette construction, mais aussi les scènes de ce qui s'y passait à l'époque romaine (maquette au 1/100e, conçue par Patrice Berger avec l'aide de J.-C. Golvin, CNRS et M. Fincker, CNRS ; réalisée par Patrice Berger).
- dans le centre de l'arène, se déroule un spectacle de gladiateurs. Ils affrontent un animal sauvage tandis que des spectateurs, répartis en groupes sur les gradins ou dans des loges, l'observent et le commentent en devisant entre eux ;

- enfin, à l'extérieur de l'amphithéâtre, le long des massives arcades, un marché s'est installé. On voit les marchands, leurs étals colorés ainsi que les tentes et les acheteurs qui marchandent, un peu comme dans un jeu de dinette.

Ici, la densité et la variété des scènes disputent les regards à l'élégance et la beauté de la reconstitution architecturale. La taille minuscule des personnages et la précision des scènes exigent que les visiteurs s'approchent pour commenter entre eux les détails qui les intéressent ou les étonnent.

En tant qu'artefact muséographique, la maquette est sans aucun doute un dispositif pertinent pour donner à voir l'une des facettes majeures du discours scientifique archéologique : la reconstitution des édifices détruits et du mode de vie d'une civilisation disparue. Il permet, non seulement, de tester la validité d'une hypothèse scientifique (comment élever et échafauder une proposition théorique vraisemblable à partir d'un ensemble hétérogène d'indices parfois ténus, discontinus et dispersés ?) mais aussi de la matérialiser concrètement comme le prouve, aujourd'hui encore, le rôle des images de synthèse pour comparer différentes variantes de reconstitution hypothétique d'un monument disparu et dont les formes architecturales sont inconnues. Et il suffit ensuite de peu de choses pour transposer ce dispositif de recherche en unité d'exposition. Pourtant, les maquettes ne sont pas nécessairement, en elles-mêmes, des dispositifs spectaculaires et attractifs. Il suffit de penser à celles des musées d'architecture (on songe, par exemple, aux élégantes et fines maquettes blanches, d'une froideur glacée, qui se trouvent à la Fondation Claude-Nicolas Ledoux, à Arc-et-Senans).

\section{Entre diorama et schématisation : une orientation cognitive}

Les maquettes, dès lors qu'elles comportent des petits personnages, se singularisent des dioramas habituellement utilisés dans les musées et les expositions. Rappelons que le diorama est un dispositif muséographique original, très élaboré et savant qui date de plus d'un siècle et demi. Démarche inverse de la présentation analytique des pièces et des objets présentés de façon isolée pour exhiber leur caractère singulier et remarquable, il s'agit, dans un diorama, 
de faire apparaître un ensemble d'ustensiles, objets et éléments mobiliers et par la même occasion de souligner les relations entre les pièces vues ailleurs comme autant de détails isolés les uns des autres.

Le diorama a été utilisé dans les musées d'ethnographie avant de conquérir les muséums d'Histoire naturelle pour répondre à la vogue de l'écologie. Un diorama est cependant spontanément lisible car le principe d'imitation et le syncrétisme que confèrent la mise en scène comme les limites matérielles du dispositif - sorte de grand volume délimité par des parois en verre (un peu comme une vitrine de magasin) - indiquent d'emblée la nature de l'activité de reconnaissance et de déchiffrage pour contempler le donné à voir ou en rechercher le sens.

Cette dimension spontanée et spectaculaire de la maquette en tant que mini diorama explique pourquoi celle-ci peut facilement devenir équivoque, voire tourner au genre spectaculaire, vidé et privé de tout contenu scientifique. Par exemple, la célèbre usine miniature animée de l'écomusée du Creusot-Montceau (étudiée par François Portet, 1992), qui fascine toujours enfants et adultes, a été promenée pendant des décennies comme une curiosité dans une baraque foraine. Et on connait, dans plusieurs pays, des parcs d'attraction, par nature populaires, qui reposent seulement sur le principe de la miniaturisation.

Ce qui fait de la maquette un dispositif non savant tient à deux principes très goûtés par les visiteurs les moins cultivés. Du principe de ressemblance (la fidélité à l'original et le réalisme dans le rendu des détails) naît une esthétique de la copie, du comme si. À cette esthétique du comme si, s'ajoute le caractère, non moins apprécié, du fait à la main, c'est-à-dire de l'hommage rendu à la minutie de cette difficile réalisation et donc à l'habileté ou à l'ingéniosité du «fabricateur».

Si l'attrait pour l'objet et le dispositif est manifeste, comment une maquette, sans rien perdre de son attractivité spontanée, peut-elle devenir un dispositif muséographique performant ? La maquette est une forme populaire. Plus encore, elle provoque une réaction presque jubilatoire en ce qu'elle évoque l'univers du jeu. Séduisante et attractive, elle suggère une posture de reconnaissance ludique et active. On l'observe, on la commente et on l'interprète comme une sorte d'énigme dans laquelle la solution est cachée. Et comme toute situation de jeu, la maquette incite à s'impliquer. Elle est en cela susceptible de devenir un support fantasmatique puisqu'elle est généralement associée, chez le visiteur adulte, aux

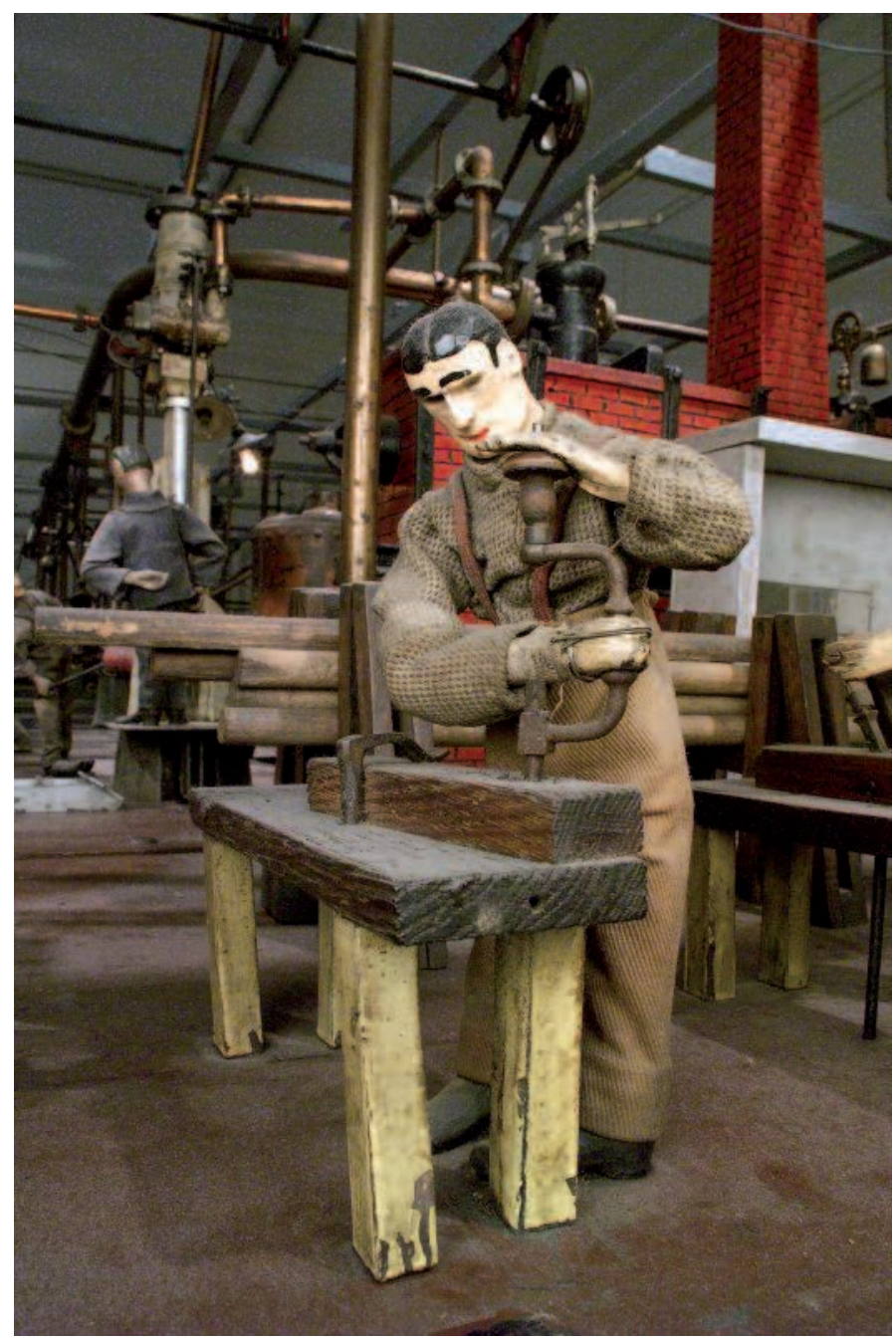

L'usine miniature : cette maquette animée des ateliers et forges du Creusot (Le menuisier, détail) réalisée entre 1890 et 1910 par Joseph Beuchot (1855-1920) mesure 2 mètres sur 5 . Joseph Beuchot, mécanicien puis contremaître aux forges de Fraisans, commence dans les années 1880 la confection de cette maquette. La grande forge, avec son marteau-pilon et ses laminoirs, la forge à main, l'atelier de menuiserie sont habités et animés par les ouvriers spécialisés dans ces diverses tâches. Cette maquette a été présentée régulièrement sur les foires et fêtes foraines avant de rejoindre les salles du musée de l'Homme et de I'Industrie (écomusée du Creusot-Montceau) au Creusot. ๑) Écomusée du Creusot-Montceau/Daniel Busseuil

souvenirs de l'enfance et à l'expérience d'une certaine liberté interprétative.

Mais surtout, contrairement à d'autres unités muséographiques froides et complexes, la maquette apparaît comme un moyen spontanément déchiffrable. Sa dimension visiblement analogique suggère qu'il suffit de la regarder comme on regarde tous les jours, comme si on était devant du réel. Elle correspond à ce que Bourdieu, à propos de la photographie, a appelé 
un art moyen. La maquette de musée est un dispositif moyen qui paradoxalement fait passer une interprétation scientifique audacieuse et raffinée pour une sorte de saynète regardée naïvement, au premier degré pour le récit qu’elle narre.

Pourtant, considérée au plan cognitif, une maquette est évidemment un outil synoptique très puissant : elle permet de donner une vue d'ensemble et de faire voir un monument comme on ne l'a jamais vu ou comme on ne peut pas le voir à pied, lors d'une visite habituelle, c'est-à-dire en cheminant dans une architecture dont les différentes salles et murs empêchent de percevoir l'ensemble et surtout l'organisation structurelle. De même, il est souvent impossible au visiteur à pied de repérer les relations d'une construction avec le contexte spatial, géographique ou urbain, sauf à prendre de la hauteur ce qui est matériellement impossible le plus souvent.

Toujours d'un point de vue cognitif, la conception d'une maquette oscille entre deux intentions contradictoires : d'une part, sa concrétude et sa fidélité (et sa ressemblance) avec le monument ou le site original, et d'autre part, son abstraction, c'est-à-dire la volonté de simplifier, d'éliminer des détails superflus ou secondaires pour la rendre plus lisible et mieux souligner ce qui, d'un point de vue scientifique ou pédagogique, est essentiel. Autrement dit, il s'agit, non seulement d'aider le visiteur à percevoir mais aussi à retenir, à mémoriser les informations essentielles. À la manière du processus de schématisation, la maquette peut devenir une contribution cognitive majeure. Dans ce cas, tout ce qui a été éliminé de la représentation (diminution de la concrétude et de l'analogie) renforce et souligne les détails conservés et ainsi mis en valeur, comme soulignés à l'intention des regardeurs. Cette maquette stylisée et plus abstraite perd en ressemblance ce qu'elle gagne en compréhension. Elle guide et modèle le regard des visiteurs et, sans le dire, impose un point de vue délibéré (Jacobi, 2005).

Ainsi, la maquette, à la manière d'un schéma, traite l'information : elle simplifie, elle stylise et unifie ou au contraire rétablit des parties manquantes. Elle oscille entre abstraction et concret. Elle met en évidence ce qui est invisible ou caché. Elle peut mettre en vedette, par contraste, ce que le maquettiste juge important. Elle propose une vue synoptique plus facile à mémoriser. Bref, c'est un puissant dispositif cognitif.

\section{Narration et reconnaissance des dispositifs spectaculaires dans les expositions archéologiques}

Nous avons souligné qu'une maquette, par la nature et l'organisation de ses constituants, se transforme en mini diorama. La présence de personnages lui confère en effet une dimension humaine. La présence d'actants, tout en favorisant l'implication, apporte toute une moisson d'informations majeures qui complète et colore la seule (fidèle) copie des éléments mobiliers et architecturaux. L'intrusion d'au moins un personnage, tout en donnant une indication d'échelle par sa taille, anime et actualise une véritable scène. Ce tableau figé, comme l'image instantanée prise dans un film ou une image dans un récit illustré est un puissant stimulant de l'imaginaire : que s'est-il passé avant ? Ou quel est l'événement susceptible de se produire?

La présence d'actants génère inévitablement une tension narrative. Ce basculement dans l'univers fictionnel est sans doute une autorisation implicite,

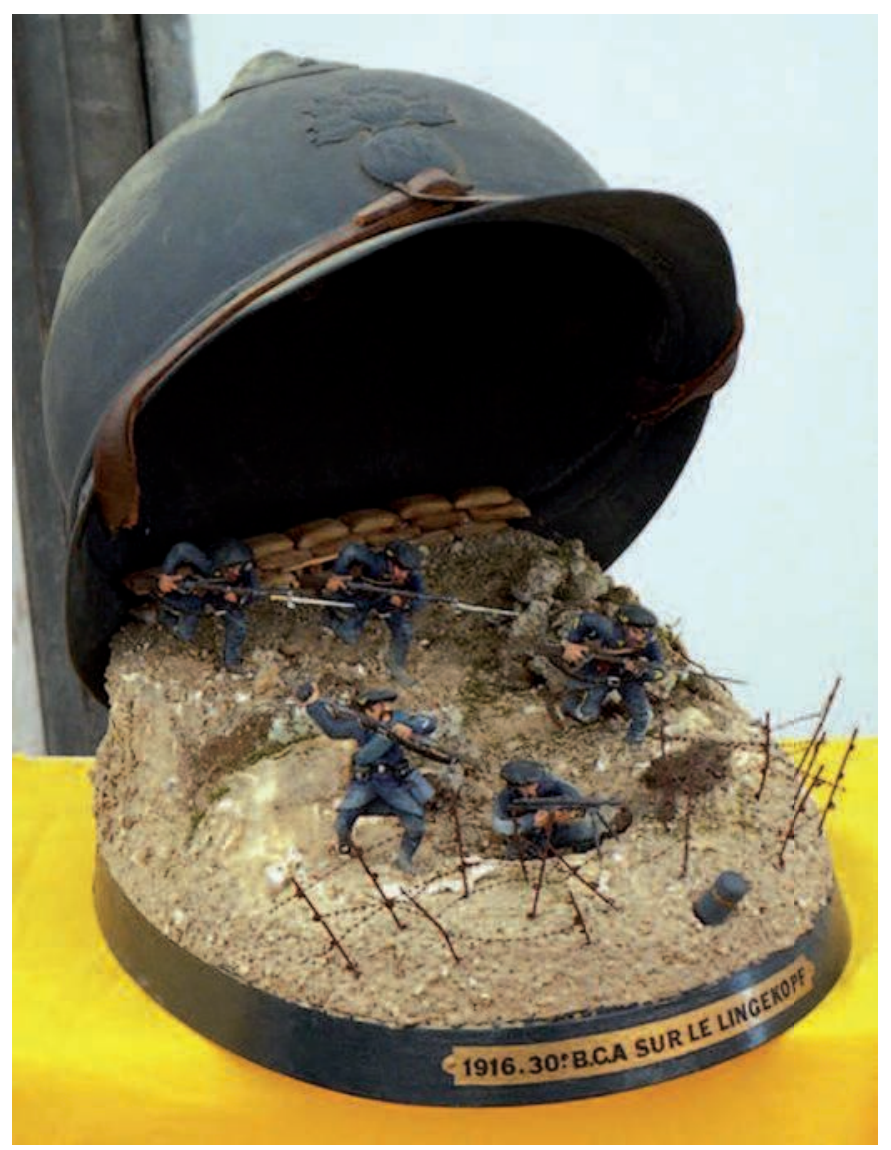

Maquette souvenir de la grande guerre. Les maquettes sont aussi des objets naïfs et populaires, réalisés avec minutie et parfois un certain talent. (C) A. Leguay et fils 
d'organiser une exposition dans une salle de la mairie tandis que le Conseil général recherchait un lieu d'implantation pour un grand musée... pendant que le scientifique, officiellement désigné par le ministère de la Culture, décidait d'interdire l'accès de la grotte au public.

Toute entreprise d'exposition met aux prises les archéologues scientifiques avec un univers différent de leur sphère professionnelle propre : celui de la communication et des médias. Les qualités scientifiques d'un chercheur professionnel (type d'investigation, nature et étendue du corpus, qualités et innovations méthodologiques, et même renouvellement et originalité des hypothèses) ne suffisent pas à faire de lui un professionnel du musée et du média exposition. Le choix de ce qui sera montré (par opposition à ce qui restera caché), l'ordre et l'arrangement des pièces, leur disposition dans l'espace ne sont pas des opérations qui obéissent obligatoirement à une logique scientifique (comme par exemple la chronologie ou la stratigraphie). Le recours à des procédés expographiques classiques ou plus modernes suppose une familiarité avec l'univers muséal. Enfin, l'écriture des textes affichés, le recours à des procédés rhétoriques métadiscursifs ne relève pas de la tradition de la rédaction du chapitre d'un catalogue scientifique ou des notices savantes habituellement commandés à des chercheurs.

Les contraintes communicationnelles propres au média exposition redonnent à tous les procédés traditionnels du récit et de l'accroche une force et une actualité insoupçonnée. C'est qu'il s'agit d'utiliser de préférence un canevas commun avec le public profane que l'on cherche à intéresser. Les maquettes réalistes et leurs analogies grotesques pimentées d'une dimension narrative sont des ressources sans égal. Chercheurs et novices n'ont que peu d'efforts à faire pour retrouver, comme dans leur enfance, un plaisir partagé : concevoir et/ou découvrir des maquettes qui, de toutes façons, ne sont que de fragiles et éphémères constructions archéologiques imaginaires.

\section{Bibliographie}

Bitgood, S. Les méthodes d'évaluation de l'efficacité des dioramas, Publics E Musées, n9, 1996, pp. 37-54.

Bourdieu, P. Un art moyen : essai sur les usages socianx de la photographie. Minuit, 1965.

Durand, G., Les structures anthropologiques de l'imaginaire ; introduction à l'archétypologie générale. Bordas, 1999.

Eidelman, J, Jacobi, D. À Chauvet, il y a tout à imaginer, in La naissance de l'art, Tallandier et La Recherche HS, 2006, pp. 155-162.

Freud, S. L'interprétation du rêve [1900]. Nouvelle traduction, PUF, 2003.

Heinich, N. et Pollak, N. Du conservateur de musée à l'auteur d'expositions : l'invention d'une position singulière, Sociologie $d u$ travail, $\mathrm{n}^{\circ} 1$, 1989.

Jacobi, D. Les faces cachées du point de vue dans les discours d'exposition, La Lettre de l'OCIM, n¹00, 2005, pp. 44-53.

Montpetit, R. Une logique d'exposition populaire : les images de la muséographie analogique, Publics \& Musées, n9, 1996, pp. 55-104.

Octobre, S. Conservateur de musée; entre profession et musée. Thèse de doctorat, EHESS, 1995.

Portet, F. L'Usine miniature: la maquette animée des usines et ateliers $d u$ Creusot, fin XIXe-début XXe siècle. Le Creusot : Écomusée, 1983 (2e édition 1992) 32 p. (tiré à part de Milieux, n¹5/16, octobre 1983-janvier 1984, pp. 16-48).

Rivière, G.-H. La muséologie selon Georges-Henri Rivière. Dunod, 1979.

Roy, J.-B. Les parcs archéologiques au risque du divertissement, Culture EMusées, n5, 2005, pp. 37-64.

Weissberg, J.-L. Le déplacement virtuel de Lascaux, Publics \& Musées, $n^{\circ} 13,1998$, pp. 129-143. 